Neurogastroenterology \& Motility

\title{
NEW MEASURES OF UPPER ESOPHAGEAL SPHINCTER DISTENSIBILITY AND OPENING PATTERNS DURING SWALLOWING IN HEALTHY SUBJECTS USING ENDOFLIP
}

\begin{tabular}{|r|l|}
\hline Journal: & Neurogastroenterology and Motility \\
\hline Manuscript ID: & Draft \\
\hline Manuscript Type: & Original Article \\
\hline Complete List of Authors: & $\begin{array}{l}\text { Regan, Julie; Adelaide and Meath Hospital, Speech \& Language Therapy; } \\
\text { Trinity College Dublin, Clinical Medicine } \\
\text { Walshe, Margaret; Trinity College Dublin, School of Clinical Speech \& } \\
\text { Language Studies } \\
\text { Rommel, Nathalie; University of Leuven, Neurosciences, ExpORL; } \\
\text { University Hospital Leuven, Neurogastroenterology Clinic } \\
\text { McMahon, Barry; Adelaide and Meath Hospital, Department of Medical } \\
\text { Physics }\end{array}$ \\
\hline Key Words: & upper esophageal sphincter, dysphagia, evaluation \\
\hline \multicolumn{2}{|c}{} \\
\hline
\end{tabular}




\title{
NEW MEASURES OF UPPER ESOPHAGEAL SPHINCTER DISTENSIBILITY AND OPENING PATTERNS DURING SWALLOWING IN HEALTHY SUBJECTS USING ENDOFLIP
}

Running Title: FLIP Evaluation of Upper Esophageal Sphincter

\author{
Julie Regan MSc BSc \\ School of Clinical Medicine, Trinity College Dublin, Ireland \\ Speech \& Language Therapy Department, Adelaide and Meath Hospital, Tallaght, Dublin 24, \\ Ireland \\ Email: reganju@tcd.ie \\ Ph.: +35314142776 \\ Fax: +35314144857
}

Margaret Walshe PhD

School of Clinical Speech \& Language Studies, Trinity College Dublin, Ireland

\section{Nathalie Rommel PhD}

Dept Neurosciences, ExpORL, University of Leuven, Belgium.

Neurogastroenterology \& Motility Clinic, University Hospital Leuven, Leuven, Belgium.

\author{
Barry P McMahon PhD \\ School of Clinical Medicine, Trinity College Dublin, Ireland \\ Medical Physics \& Clinical Engineering, Adelaide and Meath Hospital, Tallaght, Dublin 24, \\ Ireland
}




\section{Abstract}

Aims: To measure upper esophageal sphincter (UES) distensibility and extent and duration of UES opening during swallowing in healthy subjects using EndoFLIP ${ }^{\circledR}$.

Methods: Fourteen healthy subjects (20-50 years) were recruited. An EndoFLIP ${ }^{\circledR}$ probe was passed orally and the probe balloon was positioned across the UES. Two $20 \mathrm{ml}$ ramp distensions were completed and UES cross-sectional area (CSA) and intra-balloon pressure (IBP) were evaluated. At $12 \mathrm{ml}$ balloon volume, subjects completed dry, $5 \mathrm{ml}$ and $10 \mathrm{ml}$ liquid swallows and extent ( $\mathrm{mm}$ ) and duration (seconds) of UES opening and drop in IBP ( $\mathrm{mmHg}$ ) were analysed across swallows.

Results: Thirteen subjects completed the study protocol. A significant change in UES CSA $(p<.001)$ and IBP $(p<.000)$ was observed during $20 \mathrm{ml}$ distension. UES CSA increased up to $10 \mathrm{ml}$ distension $(p<.001)$, from which point IBP raised significantly $(p=0.004)$. There were significant changes in UES diameter $(\mathrm{mm})(\mathrm{p}<.000)$ and IBP $(\mathrm{mmHg})(\mathrm{p}<.000)$ during swallowing events. Resting UES diameter (4.9mm; IQR 0.02) and IBP (18.8mmHg; IQR 2.64) changed significantly during dry $(9.6 \mathrm{~mm}$; IQR $1.3: \mathrm{p}<.001)(3.6 \mathrm{mmHg} ;$ IQR 4.1 : $\mathrm{p}=0.002) ; 5 \mathrm{ml}(8.61 \mathrm{~mm} ;$ IQR 2.7: $\mathrm{p}<.001)(4.8 \mathrm{mmHg} ;$ IQR $5.7: \mathrm{p}<.001)$ and $10 \mathrm{ml}$ swallows (8.3mm; IQR 1.6: $\mathrm{p}<0.001)(3 \mathrm{mmHg} ; 4.6: \mathrm{p}<.001)$. Median duration of UES opening was 0.5 seconds across dry and liquid swallows $(p=0.91)$. Colour contour plots of EndoFLIP ${ }^{\circledR}$ data capture novel information regarding pharyngo-esophageal events during swallowing.

Conclusions: EndoFLIP ${ }^{\circledR}$ provided innovative quantitative data regarding UES distensibility and UES opening patterns during swallowing in healthy adults. This new measure of swallowing offers fresh information regarding UES dynamics which may ultimately improve patient care. 


\section{Introduction}

The upper esophageal sphincter (UES) is a muscular constriction separating the pharynx and the esophagus. It consists of the inferior pharyngeal constrictor (IPC) muscles, the cricopharyngeus (CP) and the cervical esophagus (CE), which create a 2-4 centimetre high pressure zone(1). UES high resting tone prevents diversion of air into the esophagus during inspiration and protects the airway from any retrograde passage of material refluxed from the esophagus or stomach. During swallowing, the UES needs to open adequately to ensure material passes safely and efficiently from the pharynx into the esophagus. This begins with $\mathrm{CP}$ relaxation and is followed closely by anterior and superior hyo-laryngeal excursion to stretch open the UES. Pressure from the oncoming bolus further distends the UES lumen(2).

Impaired UES opening is a feature of crico-pharyngeal dysphagia which frequently leads to tracheal aspiration and pharyngeal retention post swallow. It is commonly associated with neurological conditions (e.g. brainstem stroke, amyotrophic lateral sclerosis), myopathy (e.g. fibrosis) and structural abnormalities (e.g. Zenkers diverticulum, CP bar) (3-7). Treatment depends on the underlying cause and can include compensatory postures (e.g. head turn), rehabilitation (e.g. Shaker head lifting exercises), pharmaceutical and surgical intervention (e.g. botulinum toxin or CP myotomy)(8-11). Objective and reliable UES evaluation is critical to determine the presence and nature of UES dysfunction and to ensure the safest and most effective dysphagia intervention is provided. Currently, videofluoroscopy and pharyngeal manometry are the most commonly employed UES evaluations. However, several limitations to these evaluations have been identified including unacceptable inter-rater reliability of UES opening measures and varying resting UES pressure ranges between 35 to $200 \mathrm{mmHg}$ across studies (12-16).

The Functional Lumen Imaging Probe (FLIP) is a novel distensibility tool based on the principles of impedance planimetry(17). A balloon at the distal end of a FLIP probe is 
positioned in an anatomical lumen and is distended by filling it with a conductive solution. FLIP provides multiple CSA measures of the lumen and uses these to recreate a functional dynamic image of sphincter geometry. These CSA measures, alongside a measure of intraballoon pressure, facilitate measurement of sphincter distensibility(18). FLIP was originally designed to evaluate esopho-gastric junction (EGJ) compliance(19). It has since been employed to evaluate other anatomical sites including the sphincter of Oddi, the upper esophagus and laparoscopic lumens(20-23).

Until recently, the role of FLIP in UES evaluation had not been explored. New studies have demonstrated safe insertion and distension of the FLIP balloon in the UES of patients with dysphagia under fluoroscopy(24). The derivation of preliminary UES distensibility data and novel quantitative UES diameter and intra-balloon pressure measures by FLIP without fluoroscopy has also been reported(25). Based on these initial studies, authors hypothesise that FLIP may provide new information on UES opening characteristics during distension and may provide objective measures of UES opening during swallowing. The aims of this study were 1) to measure UES distensibility using FLIP in a group of healthy adults and 2) to quantify UES opening during dry and liquid swallowing events in this healthy group using FLIP.

\section{Materials and Methods}

\section{Subjects}

Subjects were recruited from a pool of healthy volunteers. Inclusion criteria were (1) no history of oro-pharyngeal or esophageal dysphagia, (2) no history of gastrointestinal, neurological or respiratory disease and (3) no history of head and neck cancer or ear, nose and throat conditions. Fourteen subjects (six males, eight females) with a mean age of 30 years (age range 20-50 years; $S D=11.02$ ) met inclusion criteria. Written consent was 
obtained from subjects. Ethical approval was obtained from the Research Ethics Committee, University Hospitals Leuven, Belgium.

\section{EndoFLIP® System}

A commercially developed FLIP (EndoFLIP ${ }^{\circledR}$ system, Crospon Ltd., Galway, Ireland) was used. In brief, polyurethane balloon with a maximum volume of $60 \mathrm{ml}$ is mounted on the distal $14 \mathrm{~cm}$ of a probe (EF-325) (length $240 \mathrm{~cm}$, diameter $25 \mathrm{~mm}$ ) attached to the EndoFLIP ${ }^{\circledR}$ unit. This balloon assumes a $10 \mathrm{~cm}$ long cylindrical shape with maximum diameter of $2.5 \mathrm{~cm}$ when fully filled. The maximum balloon diameter is critical to prevent airway compromise during balloon distension. Along a $7.5 \mathrm{~cm}$ segment within the balloon, 17 ring electrodes are spaced $5 \mathrm{~mm}$ apart to obtain 16 CSA measurements using an impedance planimetry technique. This allows diameter and pressure changes above (i.e. pharynx) and below (i.e. upper oesophagus) the UES to be captured and for UES opening to be observed despite its upward shift during swallowing. Excitation electrodes situated at either end of the 17 ring electrodes emit a constant low electrical current within the balloon. The probe also contains a solid-state pressure transducer to measure intra-balloon pressure.

\section{Protocol}

The EndoFLIP ${ }^{\circledR}$ system was positioned beside the subject who was seated upright on a chair within the clinic room (Neurogastroenterology \& Motility Clinic, University Hospital Leuven). The equipment was powered on and both the syringe and a pre-calibrated probe were connected to the EndoFLIP ${ }^{\circledR}$ unit. An automated purge sequence initiated by the EndoFLIP ${ }^{\circledR}$ removed air from the balloon and calibrated the pressure measurement inside. Topical anaesthesia (Lignocaine spray) was administered to the posterior pharyngeal wall and subjects were instructed to perform a dry swallow. The tip of the EndoFLIP ${ }^{\circledR}$ probe was lubricated and inserted orally by a member of the research team until the deflated balloon at 
the distal end of EndoFLIP ${ }^{\circledR}$ was judged to have passed into the proximal oesophagus ( $30 \mathrm{~cm}$ marking on EndoFLIP ${ }^{\circledR}$ catheter). The subject was transferred to a bed and seated in a 90 degree angle upright position. The EndoFLIP ${ }^{\circledR}$ catheter was held outside of the subjects' teeth by a researcher to minimize displacement during the evaluation.

\section{FIGURE 1 HERE}

When the subject became accustomed to the probe, the probe balloon within the oesophagus was distended with $10 \mathrm{mls}$ saline solution from the syringe using a touch screen function on the EndoFLIP ${ }^{\circledR}$ monitor. The inflated balloon was then slowly retracted until the hourglass shape of the UES could be visualised on the EndoFLIP ${ }^{\circledR}$ display (17-20cm marking on EndoFLIP ${ }^{\circledR}$ catheter) (Figure 1). This confirmed the balloon position in the UES. While holding the catheter in place, the balloon was deflated.

After a brief habituation period (1-2 minutes), two ramp distensions to $20 \mathrm{ml}$ were completed (rate $60 \mathrm{ml} /$ minute). Subjects were requested not to swallow during distensions and the EndoFLIP ${ }^{\circledR}$ screen was monitored to ensure the balloon remained in position. Two distensions were completed to allow for an accommodation effect. The balloon was then reinflated with a $12 \mathrm{ml}$ volume of conductive solution. Once a baseline measure of minimum UES diameter $(\mathrm{mm})$ and intra-balloon pressure $(\mathrm{mmHg})$ was recorded, subjects were asked to complete the following:

(a) two dry swallows

(b) two 5ml liquid swallow delivered orally via a syringe

(c) two $10 \mathrm{ml}$ liquid swallow delivered orally via a syringe

A minimum 10 second time period between the performances of each swallow was enforced to easily identify events during data analysis. The time (in seconds) displayed on the 
EndoFLIP ${ }^{\circledR}$ device at the execution of each swallow was recorded. When the protocol was completed, $12 \mathrm{mls}$ was deflated from the balloon and the probe was removed.

\section{Data Analysis}

\section{1) UES Distensibility}

EndoFLIP ${ }^{\circledR}$ provides sixteen measures of CSA $(\mathrm{mm} 2)$ and a measure of intra-balloon pressure $(\mathrm{mmHg})$ at a rate of ten $\mathrm{Hz}$ during distensions. Data from the second $20 \mathrm{ml}$ ramp distension was transferred from EndoFLIP ${ }^{\circledR}$ into an Excel document on a personal computer. Median CSA $\left(\mathrm{mm}^{2}\right)$ and intra-balloon pressure $(\mathrm{mmHg})$ measures and inter-quartile ranges were determined at $1,5,10,15 \& 20 \mathrm{ml}$ balloon volumes across subjects.

\section{2) Swallow Events}

EndoFLIP ${ }^{\circledR}$ measures of diameter, intra-balloon pressure and time were transferred into an Excel document. To determine change in UES opening during swallowing, three EndoFLIP ${ }^{\circledR}$ measures were selected for examination at rest and during second dry, $5 \mathrm{ml}$ and $10 \mathrm{ml}$ liquid swallow events. There were (i) extent of UES opening ( $\mathrm{mm}$ ); (ii) duration of UES opening (ms) and (iii) minimum intra-balloon pressure $(\mathrm{mmHg})$. The derivation of each variable is described below.

(i) Extent of UES Opening: EndoFLIP ${ }^{\circledR}$ provides sixteen estimated diameter $(\mathrm{mm})$ measurements (based on CSA) at a rate of ten per second throughout the examination. The minimum of the sixteen diameter measures at each time point was considered to be the narrow UES region (Figure 1B). This minimum UES diameter measure was evaluated during swallow events to ascertain the extent of UES opening during swallowing. Of note, the minimal detectable diameter of the EndoFLIP ${ }^{\circledR}$ probe is $4.8 \mathrm{~mm}$ (or $18.1 \mathrm{~mm} 2$ ) because of its physical size. 
(ii) Duration of UES opening (ms): Sixteen diameter measures were provided by EndoFLIP ${ }^{\circledR}$ at a rate of ten per second. Duration of UES opening was defined as the time from which the narrowest diameter in the UES region sharply rises from its baseline during swallowing until its return to baseline diameter (Figure 1B).

(iii) Intra-Balloon Pressure: FLIP provided ten measures of intra-balloon pressure $(\mathrm{mmHg})$ per second (Figure $1 \mathrm{~B})$. To examine change in intra-balloon pressure observed during swallow events, the minimum pressure measurement during swallowing was examined across swallows.

\section{Statistical analysis}

Data was entered into SPSS statistical software package (version 19j (IBM CORP, New York, U.S.A.). Based on Shapiro-Wilk tests, all data was not normally distributed. Data was therefore expressed as medians (interquartile range (IQR)) and non-parametric tests were employed. Kruskal-wallis tests were used to determine a change in UES CSA and intraballoon pressure across balloon volumes (1, 5, 10, 15 and 20mls) during distensibility testing. Significance was set at $\mathrm{P}<.05$. Where significance was found, multiple comparisons were made using the Wilcoxin rank sum test. Bonferroni correction was made and post-hoc tests were significant at an adjusted alpha level of 0.0127 .

Kruskal-wallis tests were also used to establish differences in UES diameter, intra-balloon pressure and duration of UES opening at baseline and across dry, $5 \mathrm{ml}$ and $10 \mathrm{ml}$ liquid swallow events. A $P<.05$ indicated statistical significance. Where significance was found, multiple comparisons were made using the Wilcoxin rank sum test. Bonferroni correction was used to determine if the post-hoc tests were significant and an adjusted alpha of 0.008 was used. 


\section{Results}

\section{1) UES Distensibility}

The EndoFLIP ${ }^{\circledR}$ probe was safely inserted and the narrowing of the UES was identified on the EndoFLIP ${ }^{\circledR}$ screen across all fourteen subjects. Thirteen of fourteen subjects completed $20 \mathrm{ml}$ ramp distensions. One subject (subject 11) was unable to tolerate more than $16 \mathrm{mls}$ in the inflated balloon in the UES for prolonged periods. The data from this subject was therefore omitted from distensibility data analysis. The second of two $20 \mathrm{ml}$ ramp distensions was included in data analysis to allow for an accommodation effect. One subject (subject 13) did not reach a maximum of $20 \mathrm{ml}$ balloon volume on their second distension (18mls) and hence their first distension (20mls) was selected for data analysis.

\section{FIGURE 2 HERE}

Across all subjects, the hourglass shape of the UES could be visualised on the EndoFLIP ${ }^{\circledR}$ screen during the ramp distension. Geometric profiles of the UES on the EndoFLIP ${ }^{\circledR}$ screen across subjects at $20 \mathrm{ml}$ balloon volume are shown in Figure 2. The minimum UES CSA increased significantly during the $20 \mathrm{ml}$ ramp distension as the balloon volume increased $(H(2)=18.32,4$ d.f., $p<.001$ ) (Figure 3). A nearly significant increase in median UES CSA was found between $1 \mathrm{~m}$ and $5 \mathrm{ml}$ balloon volumes (median CSA $18.7 \mathrm{~mm}^{2}$ and $22.5 \mathrm{~mm}^{2}$ respectively) $(p=0.028)$ and there was a significant increase in UES CSA between 5 and 10 $\mathrm{ml}$ balloon volumes (median CSA $22.5 \mathrm{~mm}^{2}$ and $23.8 \mathrm{~mm}^{2}$ respectively) $(\mathrm{p}<.001)$. The UES then resisted any further increase in CSA during the distension, as no difference in median CSA was observed between ten and fifteen mls $(p=0.382)$ or between fifteen and twenty mls $(p=0.382)$ (Figure 4).

\section{FIGURE 3 HERE}


Intra-balloon pressure also increased significantly during the $20 \mathrm{ml}$ ramp distension $(H(2)=27.36,4$ d.f., $p<.000)$ (Figure 3). No significant difference in median intra-balloon pressure was found between 1 and $5 \mathrm{mls}(p=0.463)$ or between 5 and $10 \mathrm{mls}(p<.861)$. However, once balloon inflation caused the UES CSA to reached a plateau, a significant increase in intra-balloon pressure was detected between ten and fifteen $\mathrm{mls}$ ( 4 and $13.4 \mathrm{mmHg}$ respectively $)(p=0.004)$ and between fifteen and twenty mls (13.4 and $36.9 \mathrm{mmHg}$ respectively) $(p=0.003)$ (Figure 4$)$.

\section{FIGURE 4 HERE}

\section{2) Swallow Events}

Thirteen of fourteen subjects completed the entire swallow events protocol with the distended EndoFLIP ${ }^{\circledR}$ balloon (12mls) within the UES. One subject (subject 12) could not tolerate the distended balloon in the UES for the entire protocol and was omitted from swallow manoeuvres data analysis. Data at rest and from thirty-nine swallows (the second dry, $5 \mathrm{ml} \& 10 \mathrm{ml}$ liquid swallows) within the subject group were analysed to obtain group measures of UES diameter, intra-balloon pressure \& duration of UES opening across swallow events (Table 1).

\section{TABLE 1 HERE}

There was a statistically significant change in UES diameter across swallow events $(p<.000)$. During dry swallowing, UES diameter increased from a baseline diameter measure of $4.9 \mathrm{~mm}$ to $9.6 \mathrm{~mm}$ (IQR 1.3) $(\mathrm{N}=13) \quad(\mathrm{p}<.001)$. Resting median UES diameter increased from $4.9 \mathrm{~mm}$ to $8.61 \mathrm{~mm}$ (IQR 2.7) during 5 liquid swallows $(p<.001)$. Diameter increased from $4.9 \mathrm{~mm}$ at baseline to $8.27 \mathrm{~mm}$ (IQR 1.6) during $10 \mathrm{ml}$ liquid swallows $(\mathrm{p}<.001)$. A significant median difference was also observed in UES diameter between dry and ten ml liquid swallows 
$(p<.005)$. However, no significant difference in UES diameter was observed during dry and $5 \mathrm{ml}$ swallows $(p=0.64)$ or between five and ten $\mathrm{ml}$ liquid swallows $(p=0.46)$ (Figure 5$)$.

No significant difference was evident in duration of UES opening across swallow events $(\mathrm{N}=13)(\mathrm{p}=0.91)$ (Figure 5). Median duration of UES opening remained at 0.5 seconds across subjects during dry swallowing (IQR 0.3 ), $5 \mathrm{ml}$ liquid swallows (IQR 0.3 ) and 10ml liquid swallowing (IQR 0.1) (Figure 5).

\section{FIGURE 5 HERE}

A statistically significant difference in intra-balloon pressure was observed across swallow events $(p<.000)$. Resting intra-balloon pressure dropped from 18.8 to $3.6 \mathrm{mmHg}$ (IQR 4.1 ) during dry swallowing $(p=0.002)$. Intra-balloon pressure dropped from $18.8 \mathrm{mmHg}$ at baseline to $4.8 \mathrm{mmHg}($ IQR 5.5) during $5 \mathrm{ml}$ swallows $(p<0.001)$ (Figure 5). Pressure dropped from 18.8 to $2.96 \mathrm{mmHg}(4.6)$ during $10 \mathrm{ml}$ liquid swallows $(p<0.001)$. There was no significant difference in pressure between dry and five $(p=0.6)$ or ten $\mathrm{ml}(p=0.86)$ swallows or between five and ten $\mathrm{ml}$ swallows $(p=0.35)$ (Figure 5).

\section{FIGURE 6 HERE}

EndoFLIP ${ }^{\circledR}$ diameter, pressure and time measurements are displayed in colour contour plots at rest (Figure $6 \mathrm{~A}$ ) and during swallowing (Figure $6 \mathrm{~B}$ ). Figure $6 \mathrm{~A}$ depicts the narrow band of UES (median diameter 4.9; IQR 0.02) at rest over time. At rest, the median length of the UES was $3 \mathrm{~cm}$ (IQR 1.7 ; mean $3.3 \mathrm{~cm}$ ) across subjects. As the EndoFLIP ${ }^{\circledR}$ balloon is $10 \mathrm{~cm}$ in length, an increased diameter is visible above and below the UES region, representing the pharynx and upper esophagus respectively. Median resting intra-balloon pressure is $18.8 \mathrm{mmHg}$ (IQR 2.7) over time across subjects. 
The sequence of diameter and pressure changes over time during swallowing is represented in colour contour plots (Figure 6ii). Initially, intra-balloon pressure drops from its baseline. This pressure drop is followed by a $2 \mathrm{~cm}$ upward shift of the UES, presumably caused by hyo-laryngeal excursion upon suprahyoid muscle contraction. Plots allow the position of diameter changes to be evaluated due to the $5 \mathrm{~mm}$ spacing between electrodes within the EndoFLIP ${ }^{\circledR}$ balloon. The UES lumen subsequently opens, the extent and duration of which can be measured on contour plots. At the point of UES opening, intra-balloon pressure reaches its minimum point. A narrowing is also evident at the time of swallowing in the upper esophagus, which may be indicative of esophageal peristalsis. When the UES closes, it returns back to its resting position and intra-balloon pressure increases markedly (Figure 6). These observations are evident in a common sequence across dry, $5 \mathrm{ml}$ and $10 \mathrm{ml}$ liquid swallows (Figure 7).

\section{FIGURE 7 HERE}

\section{Discussion}

In this study, distensibility and opening patterns of the UES were evaluated for the first time in a group of fourteen adult healthy subjects using EndoFLIP ${ }^{\circledR}$. EndoFLIP ${ }^{\circledR}$ was well tolerated within the UES in this subject group (thirteen of fourteen subjects) and the UES was identified across all subjects during the $20 \mathrm{ml}$ ramp distension. Major findings included the significant increase in the CSA of the UES lumen during distensibility testing. Specifically, UES CSA distended up until $10 \mathrm{ml}$ distension and then resisted further distension. This resistance was presumably due to the high resting UES tone within this healthy non-elderly subject group. From this point, intra-balloon pressure raised significantly.

This was the first study to analyse compliance of the UES lumen using EndoFLIP ${ }^{\circledR}$ in healthy adults. EndoFLIP ${ }^{\circledR}$ measurement of UES dynamics may contribute to our understanding of 
UES function and dysfunction and may, in the longer term, enhance diagnosis and hence the rehabilitative or surgical treatment of dysphagia. Further studies will determine if a distinction in UES distensibility is evident between non-elderly and elderly subjects or between healthy and clinical groups with known UES dysfunction (e.g. CP hypertonicity, CP fibrosis). No other studies have used EndoFLIP ${ }^{\circledR}$ to evaluate UES distensibility to date. As part of the validation process, measures will need to be assessed against a gold standard and checked for reliability and reproducibility.

This study also sought to examine extent and duration of UES opening during dry and liquid swallow events using EndoFLIP ${ }^{\circledR}$. This information is currently difficult to reliably quantify in clinical dysphagia practice(26). EndoFLIP ${ }^{\circledR}$ provided quantitative measures of the extent and duration of UES opening and intra-balloon pressure changes over time during dry and liquid swallowing events. Significant changes in UES diameter and intra-balloon pressure during swallowing events were found. Extent of UES opening was quantitatively measured and ranged between 8.3 and $9.6 \mathrm{~mm}$ across dry, $5 \mathrm{ml}$ and $10 \mathrm{ml}$ liquid swallows in this study. Videofluoroscopy studies have found extent of UES opening in healthy adults to be up to $0.9 \mathrm{~cm}$ during swallowing $(6,27-29)$. Duration of UES opening was 0.5 seconds across bolus volumes in this study. Measures of duration of UES opening also closely matched duration measures in previous videofluoroscopy research $(6,27,28)$. While measures of UES opening were similar to other techniques, EndoFLIP ${ }^{\circledR}$ data is not labour intensive to acquire and geometric changes in the UES during swallowing can be observed in real time on the portable EndoFLIP device as a biofeedback tool without any need for fluoroscopy.

In this study, extent of UES opening was largest for dry swallowing compared to 5 and $10 \mathrm{ml}$ liquid swallows. Intra-balloon pressure during swallowing did not decrease with increase bolus volume and duration of UES opening remained the same across dry, $5 \mathrm{ml}$ and $10 \mathrm{ml}$ liquid swallows. This lack of volume effect has also been reported in pharyngeal manometry 
studies(30). As swallow events were not randomised within the study protocol, a lack of volume effect between dry and $10 \mathrm{ml}$ liquid swallows may have been due to a fatigue element.

When diameter, pressure and time data provided by EndoFLIP ${ }^{\circledR}$ are depicted in colour contour plots, professionals are provided with an innovative graphic display of the extent and duration of UES opening on a time axis during swallowing. As detection electrodes within the probe balloon are spaced only $5 \mathrm{~mm}$ apart, EndoFLIP ${ }^{\circledR}$ can provide a rich profile of UES dynamics during swallowing and can represent the relationship between UES opening and intra-balloon pressure. Patterns regarding the sequence and duration of diameter and pressure changes were apparent across swallows in this healthy subject group and may, based on future studies, define whether bolus transport through the UES is normal or impaired. Future validation of EndoFLIP ${ }^{\circledR}$ data with measures from other physiological examinations may assist in determining if the various phases of UES opening (e.g. CP relaxation) are captured by EndoFLIP ${ }^{\circledR}$. Data in this study suggests that other pharyngoesophageal events such as the upward shift of the UES during swallowing secondary to hyolaryngeal excursion have the potential to be quantified based on these plots. This information may, in clinical practice, determine efficacy of or candidacy for rehabilitation (e.g. Shaker exercises) or surgical interventions such as botulinum toxin injections or CP myotomy (9-11).

Pharyngo-esophageal swallowing events observed in EndoFLIP ${ }^{\circledR}$ colour contour plots do present similarly to spatio-temporal pressure events on high resolution manometry (HRM) (31). The important distinction, however, is that EndoFLIP ${ }^{\circledR}$ measures changes in the narrowing of a lumen during swallowing, whereas HRM measures of UES opening are based on pressure changes during swallowing. . It is hoped that the development of new physiological gastrointestinal tests such as multi-channel intra-luminal impedance, high 
resolution manometry and EndoFLIP ${ }^{\circledR}$ may lead to better diagnostic precision and hence tailor clinical dysphagia intervention. The use of a balloon to study UES dynamics avoids the issue of pressure sensor displacement from the UES during swallowing as seen in traditional manometry.

Potential limitations to this study include the fact only ten diameter recordings are provided per second by EndoFLIP ${ }^{\circledR}$. This may have limited measures of duration of UES opening as peak values may have been missed between measurements. However, a good range of values was apparent within the subject group. These values of UES opening duration were also in keeping with previous videofluoroscopy research $(6,27,28)$.Otherwise, the minimum diameter measure provided by EndoFLIP ${ }^{\circledR}$ is $4.8 \mathrm{~mm}$, which may be narrowing some UES diameter data ranges. Perhaps one of the most important issues to consider based on this study are the optimum EndoFLIP ${ }^{\circledR}$ balloon dimensions and volumes for UES evaluation. While the study protocol was adapted from EGJ studies for UES evaluation, the probe used in these studies is a standard version for use in other regions of the esophagus. These studies suggest that EndoFLIP ${ }^{\circledR}$ balloon length and positioning need to be carefully considered during future UES testing. Care had to be taken to ensure too much of the balloon was not positioned in the pharynx during testing. Otherwise, tolerance of the balloon decreased as subjects were very sensitive to the inflated balloon in the pharynx. While a shorter probe balloon may address this issue to some extent, too short a balloon might prevent the UES opening from being captured due to its upward shift during swallowing. The effect of this can be seen most clearly on the colour contour plots where the top end of the UES can disappear during swallowing as the UES opens. Refinement of balloon dimensions and positioning during the study protocol is of prime importance to ensure that critical information is not lost during data collection.

Future research should examine UES distensibility and opening patterns in elderly healthy adults and clinical groups with known UES dysfunction (e.g. CP hypertonicity) to determine 
the effect of ageing and disease on UES compliance. It will be of interest to determine if those with electromyography (EMG)-confirmed disordered CP relaxation present differently during UES distensibility testing to those with pharyngeal phase involvement (i.e. poor hyolaryngeal excursion or weak intra-bolus pressure). Validation of EndoFLIP ${ }^{\circledR}$ against a gold standard assessment will determine the sensitivity of EndoFLIP ${ }^{\circledR}$ in diagnosing aspects of dysphagia. The development of any additional outcomes measures based on diameter, intraballoon pressure and time data obtained from EndoFLIP ${ }^{\circledR}$ needs to be explored.

\section{Conclusions}

This study presented a new technique to measure UES distensibility and to quantify the extent and duration of UES opening during swallowing events. EndoFLIP ${ }^{\circledR}$ was well tolerated by subjects and it provided valuable objective information regarding UES compliance without need for fluoroscopy. It also provided novel quantitative measures of UES opening during swallowing events which are currently lacking in clinical practice. Colour contour plots representing EndoFLIP ${ }^{\circledR}$ diameter and pressure data on a time axis provide a novel objective approach to the analysis of UES dynamics during swallowing. EndoFLIP ${ }^{\circledR}$ may provide a role in evaluating UES opening during swallowing in patients with dysphagia before and after rehabilitation or surgery. Further research is currently underway to validate EndoFLIP ${ }^{\circledR}$ as a diagnostic tool in UES evaluation.

\section{Acknowledgements \& Disclosures}

JR, MW, NR \& BM designed study. JR, MW, NR \& BM collected data. JR analysed data \& wrote paper. MW, NR \& BM reviewed paper. JR was funded by the Health Research Board, Ireland (Grant HPF/2009/39). BM previously worked as a consultant for Crospon Ltd and is currently a minor shareholder in Crospon Ltd. 


\section{References}

1. Goyal RK, Cobb BW. Motility of the pharynx, esophagus, and esophageal sphincters. Physiology of the gastrointestinal tract. 1981;1:359.

2. Cook I, Dodds W, Dantas R, Massey B, Kern M, Lang I, et al. Opening mechanisms of the human upper esophageal sphincter. American Journal of Physiology-Gastrointestinal and Liver Physiology. 1989;257(5):G748.

3. Bian RX, Choi IS, Kim JH, Han JY, Lee SG. Impaired Opening of the Upper Esophageal Sphincter in Patients with Medullary Infarctions. Dysphagia. 2009;24(2):238-45.

4. Cook I, Blumbergs P, Cash K, Jamieson G, Shearman D. Structural abnormalities of the cricopharyngeus muscle in patients with pharyngeal (Zenker's) diverticulum. Journal of gastroenterology and hepatology. 1992;7(6):556-62.

5. Murphy BA, Gilbert J, editors. Dysphagia in head and neck cancer patients treated with radiation: assessment, sequelae, and rehabilitation2009: Elsevier.

6. Leonard R, Kendall K, McKenzie S. UES opening and cricopharyngeal bar in nondysphagic elderly and nonelderly adults. Dysphagia. 2004;19(3):182-91.

7. Takasaki K, Umeki H, Enatsu K, Kumagami H, Takahashi H. Evaluation of swallowing pressure in a patient with amyotrophic lateral sclerosis before and after cricopharyngeal myotomy using high-resolution manometry system. Auris Nasus Larynx. 2010;37(5):644-7.

8. McCulloch TM, Hoffman MR, Ciucci MR. High-Resolution Manometry of Pharyngeal Swallow Pressure Events Associated With Head Turn and Chin Tuck. The Annals of otology, rhinology \& laryngology. 2010;119(6):369-76.

9. Shaker R, Easterling C, Kern M, Nitschke T, Massey B, Daniels S, et al. Rehabilitation of swallowing by exercise in tube-fed patients with pharyngeal dysphagia secondary to abnormal UES opening*. Gastroenterology. 2002;122(5):1314-21.

10. Kos MP, David EF, Klinkenberg-Knol EC, Mahieu HF. Long-Term Results of External Upper Esophageal Sphincter Myotomy for Oropharyngeal Dysphagia. Dysphagia. 2010:1-8.

11. Alfonsi E, Merlo IM, Ponzio M, Montomoli C, Tassorelli C, Biancardi C, et al. An electrophysiological approach to the diagnosis of neurogenic dysphagia: implications for botulinum toxin treatment. Journal of Neurology, Neurosurgery \& Psychiatry. 2010;81(1):54.

12. Stoeckli SJ, Huisman TAGM, Seifert BAGM, Martin-Harris BJW. Interrater reliability of videofluoroscopic swallow evaluation. Dysphagia. 2003;18(1):53-7.

13. Kuhlemeier $K$, Yates $P$, Palmer J. Intra-and interrater variation in the evaluation of videofluorographic swallowing studies. Dysphagia. 1998;13(3):142-7. 
14. McCullough GH, Wertz RT, Rosenbek JC, Mills RH, Webb WG, Ross KB. Inter-and intrajudge reliability for videofluoroscopic swallowing evaluation measures. Dysphagia. 2001;16(2):110-8.

15. Pandolfino JE, Kahrilas PJ. AGA technical review on the clinical use of esophageal manometry. Gastroenterology. 2005;128(1):209-24.

16. Sivarao D, Goyal RK. Functional anatomy and physiology of the upper esophageal sphincter* 1. The American journal of medicine. 2000;108(4):27-37.

17. McMahon B, Frøkjær JB, Drewes AM, Gregersen H. A new measurement of oesophago-gastric junction competence. Neurogastroenterology \& Motility. 2004;16(5):5436.

18. Fox M, Sweis R. Future directions in esophageal motility and function-new technology and methodology. Neurogastroenterology \& Motility. 2012;24:48-56.

19. McMahon BP, Frøkjær JB, Kunwald P, Liao D, Funch-Jensen P, Drewes AM, et al. The functional lumen imaging probe (FLIP) for evaluation of the esophagogastric junction. American Journal of Physiology-Gastrointestinal and Liver Physiology. 2007;292(1):G377.

20. Kunwald P, Drewes A, Kjær D, Gravesen FH, Mcmahon B, Madácsy L, et al. A new distensibility technique to measure sphincter of Oddi function. Neurogastroenterology \& Motility. 2010;22(9):978-e253.

21. Kwiatek MA, Hirano I, Kahrilas PJ, Rothe J, Luger D, Pandolfino JE. Mechanical properties of the esophagus in eosinophilic esophagitis. Gastroenterology. 2010.

22. SCHWANNOMA LROFAP. 2010 Scientific Session of the Society of American Gastrointestinal and Endoscopic Surgeons (SAGES) National Harbor, Maryland, USA, 14-17 April 2010 Video Presentations. Surg Endosc. 2010;24:S270-S97.

23. Perretta S, Dallemagne B, McMahon B, D'Agostino J, Marescaux J. Improving functional esophageal surgery with a "smart" bougie: endoflip. Surgical Endoscopy. 2011:1-.

24. Regan J, McMahon BP. T1907 A Novel Distensibility Technique for Measuring Upper Esophageal Function-Pilot Data. Gastroenterology. 2010;138(5):S-604-S-.

25. Regan J, Walshe, M., Rommel, N. \& McMahon, B. P. A New Evaluation of the Upper Esophageal Sphincter Using the Functional Lumen Imaging Probe- A Preliminary Report. Diseases of the Esophagus. 2012 (accepted).

26. Regan J, Walshe M, McMahon BP. Current evaluation of upper oesophageal sphincter opening in dysphagia practice: an international SLT survey. International Journal of Language \& Communication Disorders. 2011. 
27. Martin-Harris B, Brodsky MB, Price CC, Michel Y, Walters B. Temporal coordination of pharyngeal and laryngeal dynamics with breathing during swallowing: single liquid swallows. Journal of Applied Physiology. 2003;94(5):1735.

28. Rofes L, Arreola V, Romea M, Palomera E, Almirall J, Cabré M, et al. Pathophysiology of oropharyngeal dysphagia in the frail elderly. Neurogastroenterology \& Motility. 2010;22(8):851-e230.

29. Logemann JA, Pauloski BR, Rademaker AW, Kahrilas PJ. Oropharyngeal swallow in younger and older women: videofluoroscopic analysis. Journal of Speech, Language, and Hearing Research. 2002;45(3):434.

30. Butler SG, Stuart A, Castell D, Russell GB, Koch K, Kemp S. Effects of age, gender, bolus condition, viscosity, and volume on pharyngeal and upper esophageal sphincter pressure and temporal measurements during swallowing. Journal of Speech, Language, and Hearing Research. 2009;52(1):240.

31. Hoffman MR, Ciucci MR, Mielens JD, Jiang JJ, McCulloch TM. Pharyngeal swallow adaptations to bolus volume measured with high resolution manometry. The Laryngoscope. 2010;120(12):2367-73. 


\section{Tables}

Table 1. Diameter, Pressure and Duration of UES Opening Measures At Baseline and During Dry, $5 \mathrm{ml}$ and $10 \mathrm{ml}$ Liquid Swallow Events (12ml Balloon Volume) $(\mathbf{N}=13)$

\begin{tabular}{|c|c|c|c|c|c|c|c|c|c|c|c|}
\hline \multirow[b]{2}{*}{ No. } & \multicolumn{4}{|c|}{$\begin{array}{c}\text { Minimum UES Diameter } \\
(\mathbf{m m})\end{array}$} & \multicolumn{4}{|c|}{$\begin{array}{l}\text { Minimum Intra-Balloon } \\
\text { Pressure (mmHg) }\end{array}$} & \multicolumn{3}{|c|}{$\begin{array}{c}\text { UES Opening } \\
\text { Duration (secs) }\end{array}$} \\
\hline & base & dry & $\begin{array}{c}5 \mathrm{ml} \\
\text { liquid }\end{array}$ & $\begin{array}{l}\text { 10ml } \\
\text { liquid }\end{array}$ & base & dry & $\begin{array}{l}5 \mathrm{ml} \\
\text { liquid }\end{array}$ & $\begin{array}{l}\text { 10ml } \\
\text { liquid }\end{array}$ & dry & $\begin{array}{c}5 \mathrm{ml} \\
\text { liquid }\end{array}$ & $\begin{array}{l}\text { 10ml } \\
\text { liquid }\end{array}$ \\
\hline 1 & 4.88 & 10.26 & 7.2 & 8.27 & 18.69 & 6.55 & 6.64 & 2.96 & 0.7 & 0.5 & 0.5 \\
\hline 2 & 4.9 & 9.7 & 7.9 & 8.53 & 14.97 & 20.19 & 12.14 & 10.4 & 0.4 & 0.6 & 0.5 \\
\hline 3 & 4.86 & 9.46 & 8.61 & 7.91 & 18.54 & 1.62 & 4.46 & 2.82 & 0.3 & 0.6 & 0.5 \\
\hline 4 & 4.89 & 9.63 & 8.62 & 8.92 & 26.24 & 1.71 & 8.12 & 9.17 & 0.6 & 0.3 & 0.5 \\
\hline 5 & 4.96 & 8.8 & 8.75 & 7.62 & 19.31 & 5.08 & 7.17 & 7.06 & 0.3 & 0.3 & 0.4 \\
\hline 6 & 4.9 & 8.87 & 9.95 & 9.02 & 18.85 & 3.6 & 7.07 & 6.66 & 0.5 & 0.4 & 0.2 \\
\hline 7 & 4.87 & 9.53 & 6.25 & 10.02 & 18.84 & -0.02 & -1.4 & 0.35 & 0.4 & 0.5 & 0.5 \\
\hline 8 & 4.87 & 9.55 & 10.15 & 7.95 & 18.62 & 2.1 & 3.36 & 2.64 & 0.4 & 0.3 & 0.5 \\
\hline 9 & 4.94 & 7.58 & 7.3 & 7.06 & 19.16 & 7.48 & 4.75 & 1.84 & 0.3 & 0.6 & 0.6 \\
\hline 10 & 4.89 & 10.84 & 10.48 & 6.05 & 19.58 & 4.68 & 4.32 & 5.78 & 0.7 & 0.6 & 0.5 \\
\hline 11 & 4.9 & 9.99 & 8.12 & 9 & 31.01 & -1.93 & -0.23 & -2.99 & 0.7 & 0.7 & 0.5 \\
\hline 13 & 4.88 & 10.93 & 6.5 & 6.89 & 14.82 & 2.78 & 7.09 & 5.64 & 0.5 & 0.5 & 0.4 \\
\hline 14 & 4.88 & 8.49 & 9.85 & 8.41 & 15.07 & 4.29 & -3.73 & 2.8 & 0.6 & 0.6 & 0.7 \\
\hline
\end{tabular}




\section{Figure Legends}

Figure 1. Positioning of EndoFLIP ${ }^{\circledR}$ Balloon in UES of Healthy Subjects

Figure 2. Geometric Profiles of UES on EndoFLIP ${ }^{\circledR}$ Screen at $20 \mathrm{ml}$ Balloon Volume during Distensibility Testing $(\mathbf{N}=13)$

Figure 3. UES Distensibility during 20ml Ramp Distension ( $N=13$ )

Figure 4. Median CSA \& Intra-Balloon Pressure Changes during 20ml Ramp Distension Across Subjects ( $N=13)$

Figure 5. UES Diameter, Intra-Balloon Pressure and Duration of UES Opening At Rest and during Dry, $5 \mathrm{ml} \& 10 \mathrm{ml}$ Liquid Swallows $(\mathrm{N}=13)$

Figure 6. Colour Contour Plots of EndoFLIP ${ }^{\circledR}$ Diameter, Intra-Balloon Pressure and Time data at rest (i) and during swallowing (ii).

In Figure 6i, colour contour plots depict diameter and intra-balloon pressure changes over time. Time is on the $x$-axis and sixteen diameter measurements from seventeen detection electrodes spaced $5 \mathrm{~mm}$ apart within the within the EndoFLIP balloon are displayed on the $y$ axis. Each diameter measure is assigned a colour (see legend). The narrowest diameter measures (in red) are at the level of the UES. The black line represents intra-balloon pressure over time.

In Figure 6ii, $(A)$ resting UES diameter is $4.9 \mathrm{~mm}$ and the resting length of the UES is $4 \mathrm{~cm}$. As (B) the swallow is elicited, (C) a drop in intra-balloon pressure shortly precedes (D) a $2 \mathrm{~cm}$ upward shift of the UES, presumably due to hyo-laryngeal excursion. (E) The UES diameter then increases to $9 \mathrm{~mm}$ during swallowing. (F) A narrowing within the upper esophagus, perhaps due to peristalsis, is observed at the point of UES opening. (G) The UES then closes and $(\mathrm{H})$ intra-balloon pressure increases markedly. (I) The UES returns to its resting position and $(\mathrm{J})$ intra-balloon pressure gradually decreases.

Figure 7. Colour Contour Plots of EndoFLIP ${ }^{\circledR}$ Diameter, Intra-Balloon Pressure and Time Measures during Dry, $5 \mathrm{ml}$ and $10 \mathrm{ml}$ Liquid Swallows 

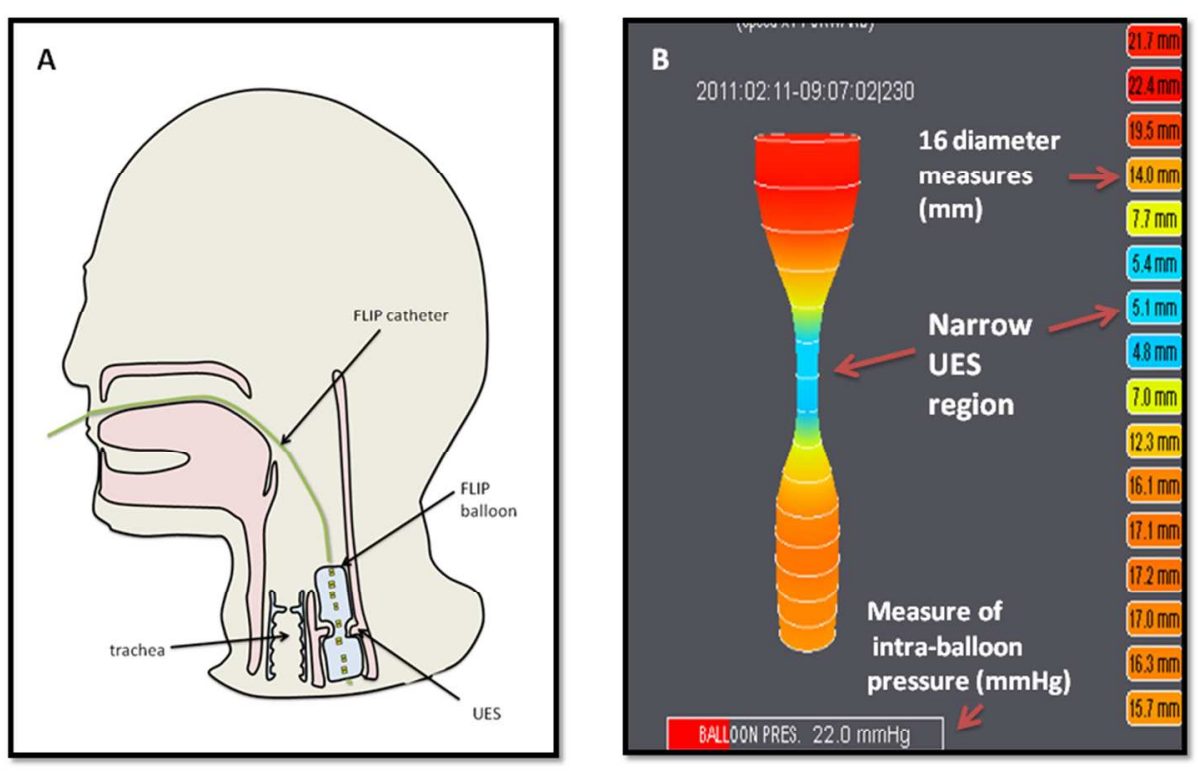

Positioning of EndoFLIP Balloon in UES of Healthy Subjects $254 \times 190 \mathrm{~mm}(96 \times 96 \mathrm{DPI})$ 


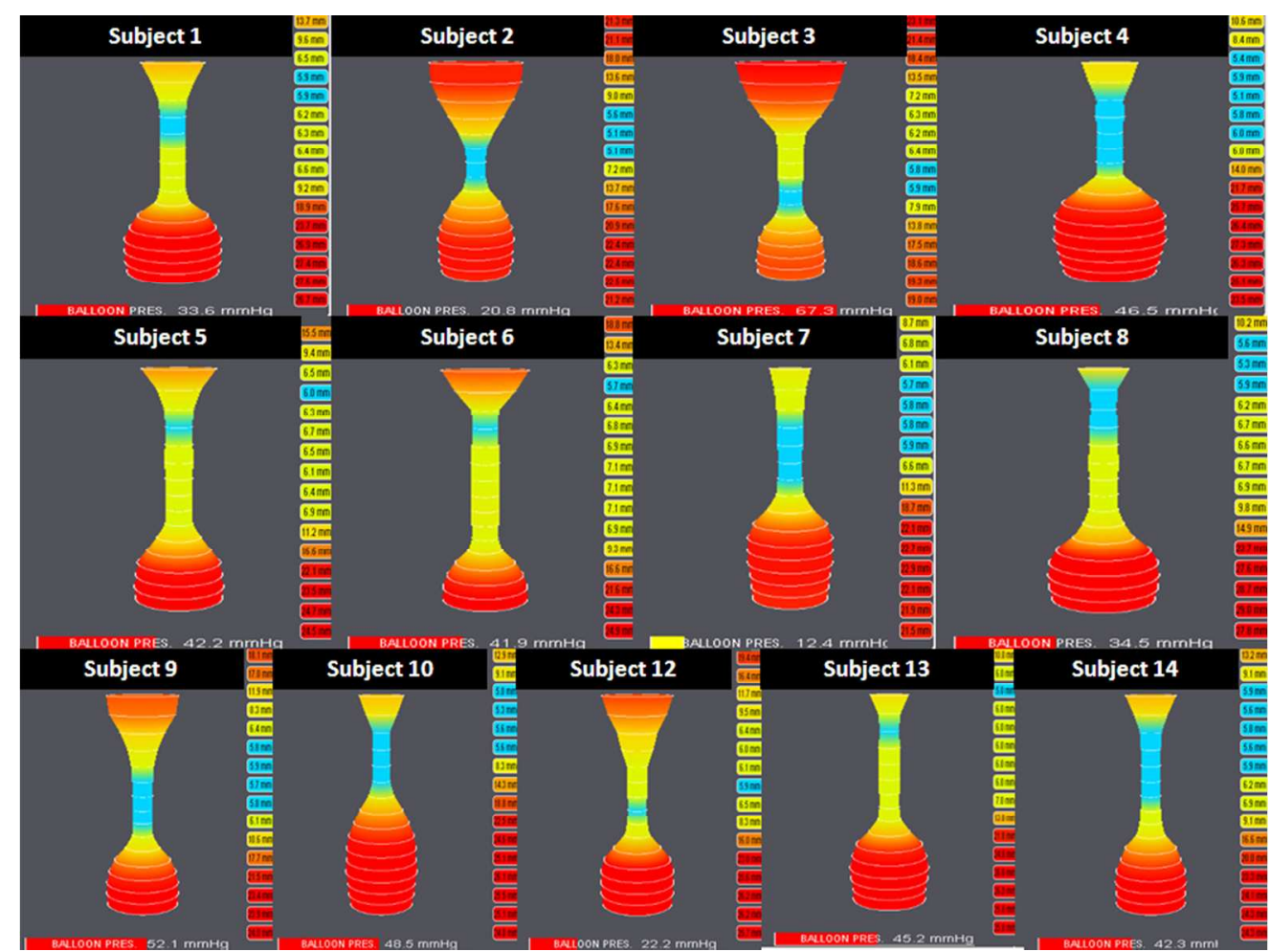
Geometric Profiles of UES on EndoFLIP $®$ Screen at $20 \mathrm{ml}$ Balloon Volume during Distensibility Testing $(\mathrm{N}=13)$
$254 \times 190 \mathrm{~mm}(96 \times 96 \mathrm{DPI})$


Neurogastroenterology and Motility

Page 24 of 28

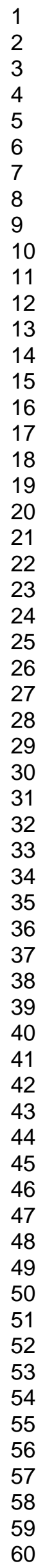



UES Distensibility during $20 \mathrm{ml}$ Ramp Distension ( $\mathrm{N}=13$ ) $254 \times 190 \mathrm{~mm}(96 \times 96 \mathrm{DPI})$ 

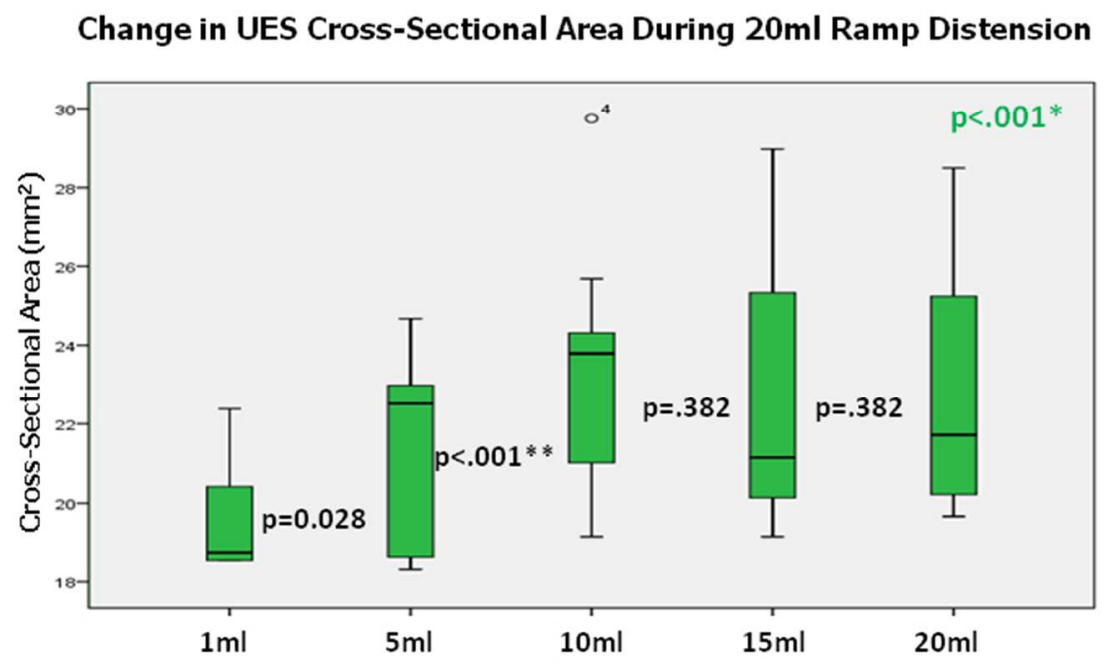

Change in Intra-Balloon Pressure During 20ml Ramp Distension

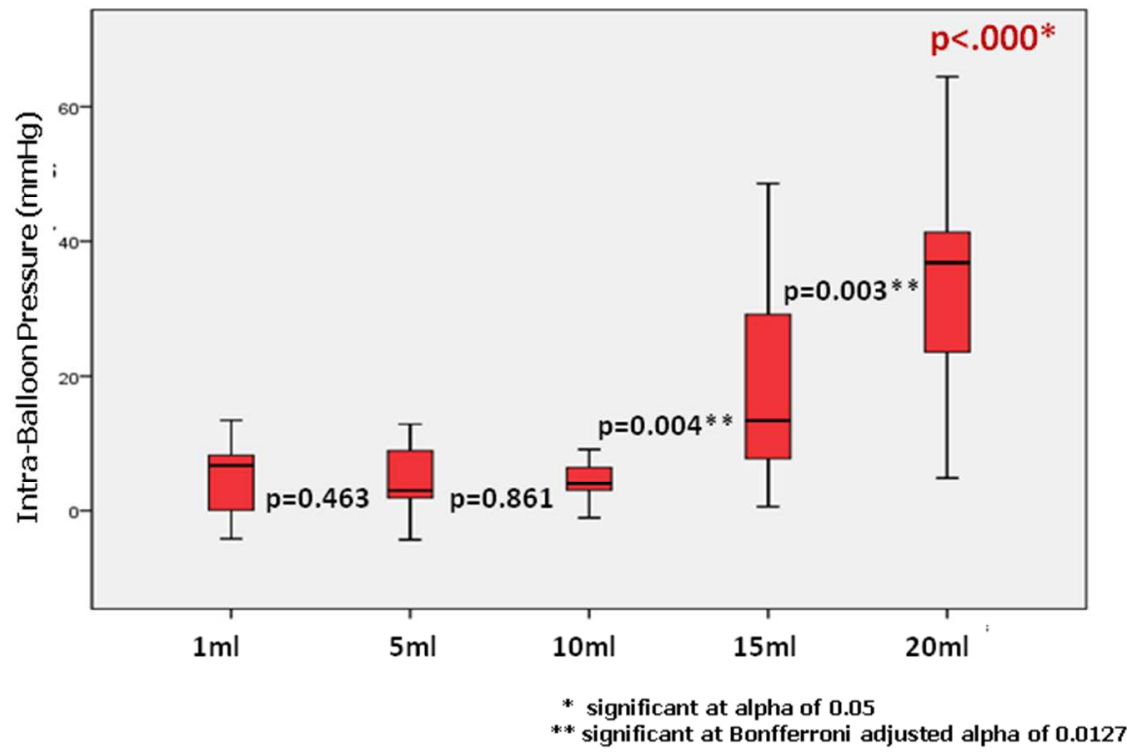

Median CSA and Intra-Balloon Pressure Changes during 20ml Ramp Distension Across Subjects $(\mathrm{N}=13)$ $190 \times 254 \mathrm{~mm}(96 \times 96 \mathrm{DPI})$ 



Duration of UES Opening Across Swallow Events $(\mathrm{N}=13)$

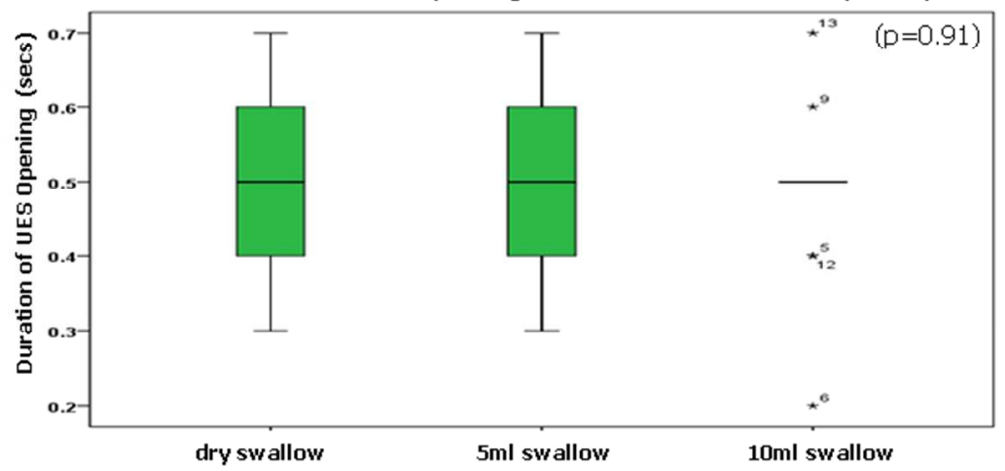

UES Diameter, Intra-Balloon Pressure and Duration of UES Opening (i) At Rest and (ii) during $5 \mathrm{ml}$ and $10 \mathrm{ml}$ Liquid Swallowing $190 \times 254 \mathrm{~mm}(96 \times 96$ DPI) 

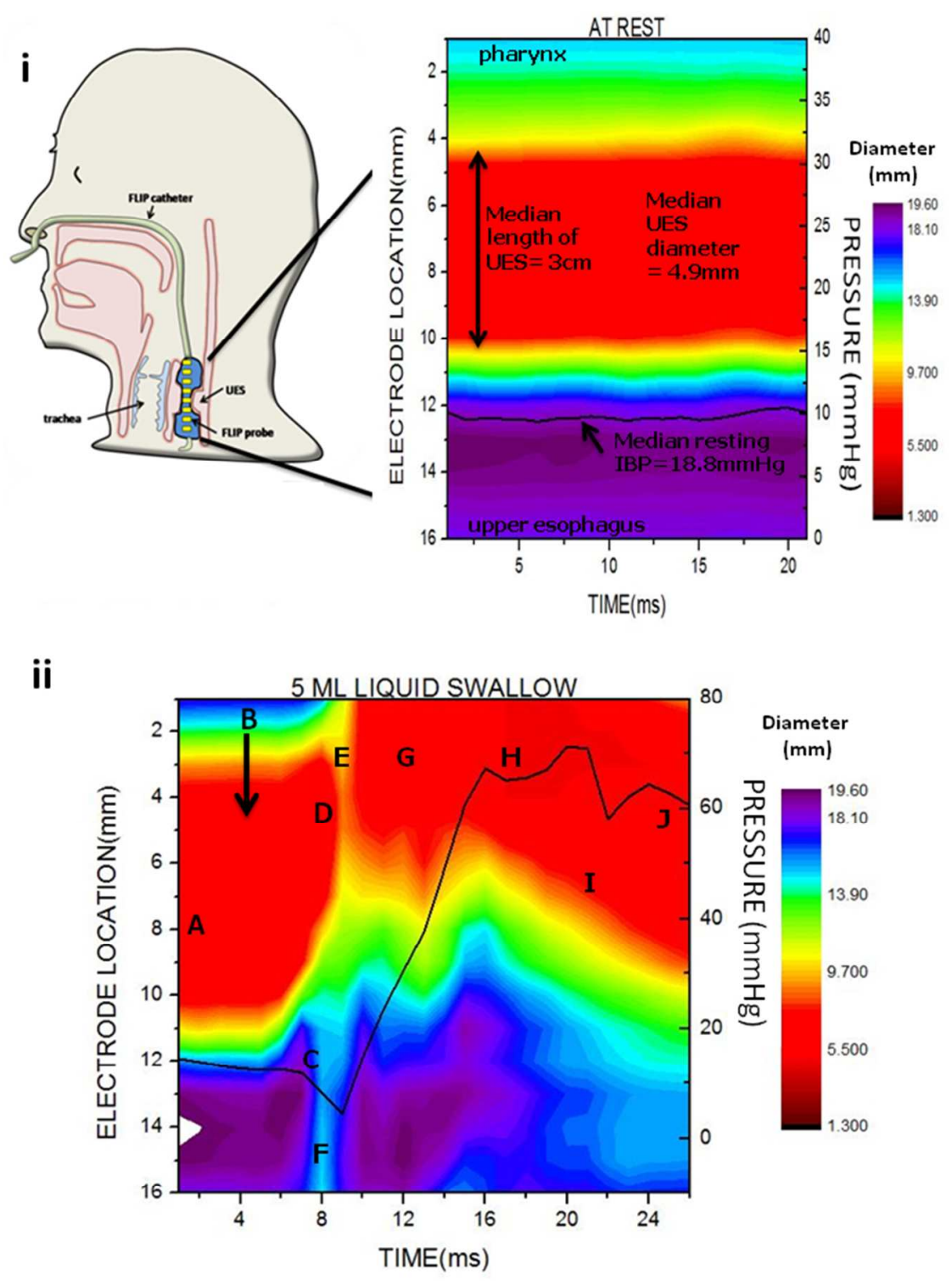

In Figure 6i, colour contour plots depict diameter and intra-balloon pressure changes over time. Time is on the $\mathrm{x}$-axis and sixteen diameter measurements from seventeen detection electrodes spaced $5 \mathrm{~mm}$ apart within the EndoFLIP balloon are displayed on the $y$-axis. Each diameter measure is assigned a colour (see legend). The narrowest diameter measures (in red) are at the level of the UES. The black line represents intra-balloon pressure over time.

In Figure 6ii, resting UES diameter is $4.9 \mathrm{~mm}$ and the resting length of the UES is $4 \mathrm{~cm}$. As (B) the swallow is elicited, (C) a drop in intra-balloon pressure shortly precedes (D) a $2 \mathrm{~cm}$ upward shift of the UES, presumably due to hyo-laryngeal excursion. (E) The UES diameter then increases to $9 \mathrm{~mm}$ during swallowing. (F) A narrowing within the upper esophagus, perhaps due to peristalsis, is observed at the point of UES opening. (G) The UES then closes and $(\mathrm{H})$ intra-balloon pressure increases markdely. (I) The UES returns to its resting position and $(\mathrm{J})$ intra-balloon pressure gradually decreases. 

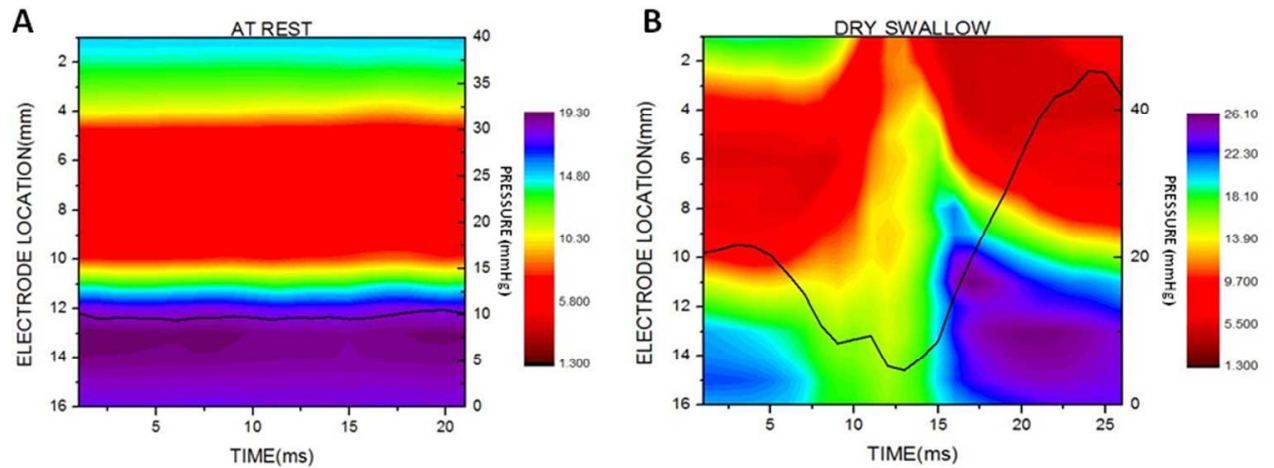

C
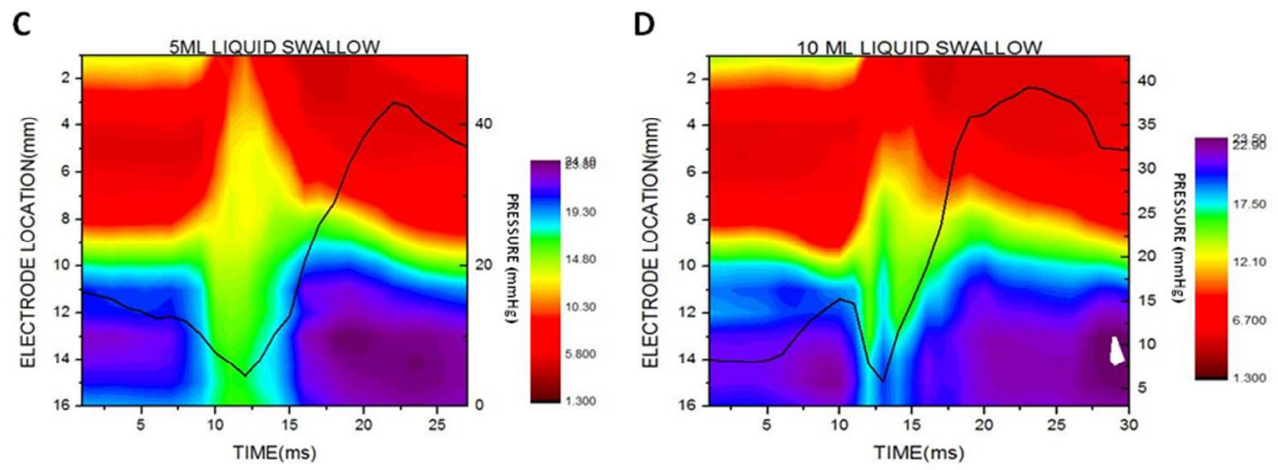

Colour Contour Plots of EndoFLIP Diameter, Intra-Balloon Pressure and Time Measures during Dry, 5ml and $10 \mathrm{ml}$ Liquid Swallows $254 \times 190 \mathrm{~mm}(96 \times 96$ DPI) 